\title{
A Study of Halo Naevi, Vitiligo and Asymptomatic Autoimmune Thyroiditis
}

\author{
${ }^{1}$ Department of Dermatology, Faculty of Medicine and Pharmacy, Dunarea de Jos University, Galati \\ ${ }^{2}$ Department of Dermatology, Elias Emergency Hospital, Bucharest, Romania \\ ${ }^{3}$ Dermatology Outpatient Clinic, University Hospital "Saint-Louis", Paris, France \\ ${ }^{4}$ Carol Davila University and Central Reference Laboratory Synevo, Bucharest, Romania
}

Alin Laurentiu Tatu ${ }^{1 *}$, Victor Gabriel Clatici ${ }^{2}$, Marius-Anton Ionescu ${ }^{3}$, Violeta Corina Cristea ${ }^{4}$

*Corresponding author: Tatu Alin Laurentiu, Department of Dermatology, Faculty of Medicine and Pharmacy, Dunarea de Jos University, Al.I. Cuza street no 39, Galati, Postal code-800101, Romania, Tel: 00-40-728267435; Fax: 00-40-236-415705;

E-mail: dralin_tatu@yahoo.com,dr.toni.ionescu@gmail.com

Citation: Tatu, A.L, et al. A Study of Halo Naevi, Vitiligo and Asymptomatic Autoimmune Thyroiditis. (2017) Invest Dermatol Venereol Res 3(1): 105- 107.

Keywords: Halo naevi; Dermoscopy; Vitiligo; Anti-thyroid peroxidase; Autoimmune thyroiditis
Received date: March 23, 2017

Accepted date: April 05, 2017

Published date: April 11, 2017

DOI: $10.15436 / 2381-0858.17 .1438$

\section{Background}

Halo Naevus (HN) is a dermatologic condition characterized by a typical whitish rim arround an existing melanocytic congenital or acquired nevus, it was first described in 1916 by Sutton ${ }^{[1]}$. Halo nævi are common in children and young adults, with a mean age at onset of 15 years. The incidence in general population is estimated to be approximately $1 \%{ }^{[2]}$. Affected individuals frequently have multiple lesions which are usually localized on the back. $\mathrm{HN}$ is an autoimmune response and $\mathrm{T}$ lymphocytes are considered to play a key role in the progressive destruction of nævus' cells. Multiple HN is rather rare and this could suggest a higher risk of vitiligo or of autoimmune disease development comparing to solitary $\mathrm{HN}$ lesion risks. The mechanism of halo phenomenon is still non completely defined and is suggested to be induced by a destruction of melanocytes by immune response of cytotoxic $\mathrm{T}$ cells ${ }^{[3]}$ or due to IgM autoantibodies induction $^{[4]}$.

\section{Purpose}

We sought to assess the association of Hallo Naevi (HN) or of HN-vitiligo with thyroiditis by screening markers of thyroiditis in patients presenting Hallo Naevi (HN) and HN associated with vitiligo.

\section{Materials and Methods}

In this study were included adults patients with halo nævus and / or vitiligo presented in dermatology outpatient clin- ic between 2010 and 2016. Clinical and dermoscopy examination were performed. The possible association between vitiligo and thyroiditis was assessed by peripheral blood dosage of Anti-Thyroid Peroxidase (ATPO).

\section{Results}

Were included 37 patients, 16 female and 21 male. Twenty five patients $(67.56 \%)$ had one $\mathrm{HN}, 7$ patients $(18.91 \%)$ had $2 \mathrm{HN}$ and $5(15.31 \%)$ of the patients had $3 \mathrm{HN}$. From 37 patients with halo nevus $10(27.02 \%)$ had associated vitiligo. In all cases vitiligo occurred 3 - 4 years after the onset of HN. In all 10 patients with $\mathrm{HN}$ and associated vitiligo, lesions were of non-segmental type: limited, symmetric, small and large lesions of extremities. Among them five had one HN (4 female and 1 male), 2 patients had $2 \mathrm{HN}$ (one male and one female), 3 patients had $3 \mathrm{HN}$.

In 27 (72.98\%) patients with HN ( and no vitiligo): ATPO peripheral blood levels were normal in 26 patients (96.29\%), a 13 years old boy had high ATPO (presented in the discussion) (3.71\%).

In 10 patients with $\mathrm{HN}$ and associated vitiligo: 3 had high ATPO results $(33.33 \%$ of patients, 1 male with $2 \mathrm{HN}$ and 2 female with $3 \mathrm{HN}$ ) with a $2 / 1$ female/male ratio. One of female with $3 \mathrm{HN}$ had a history of autoimmune thyroiditis and vitiligo - both occurred after the onset of HN (by order thyroiditis, HN then vitiligo). In 2 others cases with high ATPO clinical appear- 
ance of the conditions was HN, vitiligo and thyroiditis. Vitiligo features were in all cases of type non-segmental with bilateral hypo-chromic, symmetrical small macules $(0.3-0.8 \mathrm{~cm}$ diameter) and Koebner phenomena or large plaques located on extremities (Figure 1).

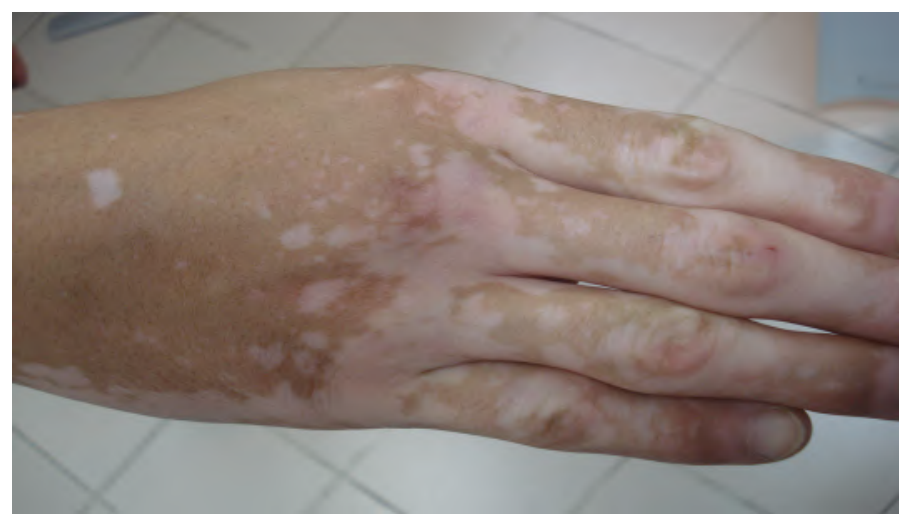

Figure 1: Vitiligo of the hand.

\section{Discussion}

Thirty eight patients were initially included. One patient was excluded as he presented one lesion that was not a halo naevus but a melanoma with peripheral regression send to surgery and oncology. Vitiligo and halo naevi are both pigmentary disorders of the skin characterized by the acquired loss of functional epidermal melanocytes manifesting as white macules and patches. The cellular mechanisms and biochemical changes that result in the appearance of these two types of achromic lesions and the direct relationship between vitiligo and halo naevi are still subject of discussion. The tendency of different morphologic alterations in mitochondria from perilesional vitiligo skin and from perilesional halo nevi skin reflect heterogeneous backgrounds between the two diseases, revealing that vitiligo and halo naevi may have separate pathogenic mechanisms.

There is strong evidence that immunological mechanisms are at the base of melanocytic destruction: the presence progressive destruction of of an inflammatory lymphocytic infiltrate that leads to progressive destruction of naevi cells; the presence of antibodies against nevi cells ${ }^{[5]}$. Recent studies highlighted similarities and differences between events that occur in regression of melanoma, vitiligo and in Sutton's phenomenon $^{[6-8]}$.

The association of vitiligo with autoimmune thyroid diseases and the increased prevalence of autoantibodies including thyroid autoantibodies in vitiligo had been proven by several recent studies ${ }^{[9-11]}$. In Sutton's nevi was usually described an inflammatory cellular infiltrate surrounding symmetrically the naevus, consisting mainly of T-lymphocytes. B-lymphocytes, Langerhans cells and macrophages are absent or rarely observed $^{[3]}$. Thyroiditis is defined histologically by the presence of a diffuse lymphocytic infiltrate in the thyroid gland, with the formation of lymphoid follicles. Cell mediated and antibody mediated immune responses lead to the destruction of thyroid cells $^{[10]}$. In patients with multiple $H N$, the risk of vitiligo and other autoimmune diseases seems to be higher than in pediatric patients with a single $\mathrm{HN}$. The female/male ratio is $5-10 / 1$ in autoimmune thyroiditis and the onset of disorder usually takes place between the third and sixth decade of life. Due the fact that halo naevus' onset is usually be earlier than vitiligo, clinicians should pay particular attention even to patients presenting children/boys with one or several HN. The follow-up of $\mathrm{HN}$ is clinical and by dermoscopy, very high photo-protection is necessary (SPF 50+) for HN of photo exposed areas. Non-segmental, stable, limited vitiligo management is most frequently made by potent topical steroid therapy, topical calcineurin inhibitors, or narrow-band UVB $311 \mathrm{~nm}$ or associations phototherapy by UVA and psoralen (PUVA) (phototherapy is contraindicated in areas where vitiligo is associated with $\mathrm{HN}$ ), excimer laser $308 \mathrm{~nm}$.

A 13 years old boy with congenital melanocitic naevi that was referred to dermatology presented a recent white patch arround one naevus. Clinical evaluation revealed a whitish rim arround an congenital melanocitic nevus from the lumbar area, without cutaneous induration, resembling a halo nevus (Figure 2).

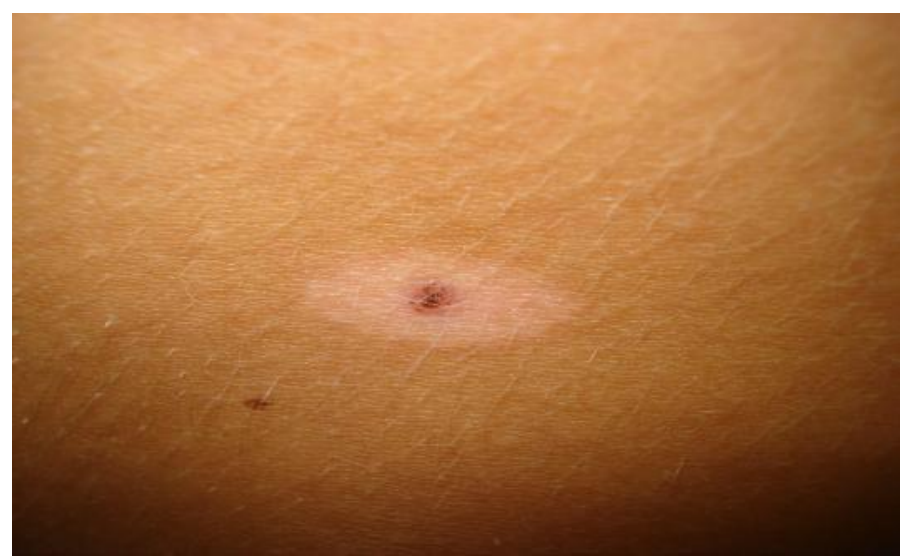

Figure 2: Halo nevus. Clinical image.

Dermoscopy revealed a mixed pattern composed of a central network, structureless light brown-gray area and some small brown globules in periphery (Figure 3$)^{[12]}$. There was no family or personal history of vitiligo or autoimune diseases. Anti-Thyroid Peroxidase (ATPO) levels were 107.6UI/mL (normal values 0 - 34UI/mL), Thyroid Stimulating Hormone (TSH) was $3,2 \mathrm{uUI} / \mathrm{mL}$ (normal values $0,4-4 \mathrm{uUI} / \mathrm{mL}$ ), free thyroxine (fT4) was $1,14 \mathrm{ng} / \mathrm{dL}$ (normal values $0,98-1,63 \mathrm{ng} / \mathrm{dL}$ ). Thyroid ultrasound showed heterogeneous thyroid echo texture. Anti-thyroglobulin and fT4 were not performed but the patient was refered to the endocrinologist for specialty investigations and monitoring.

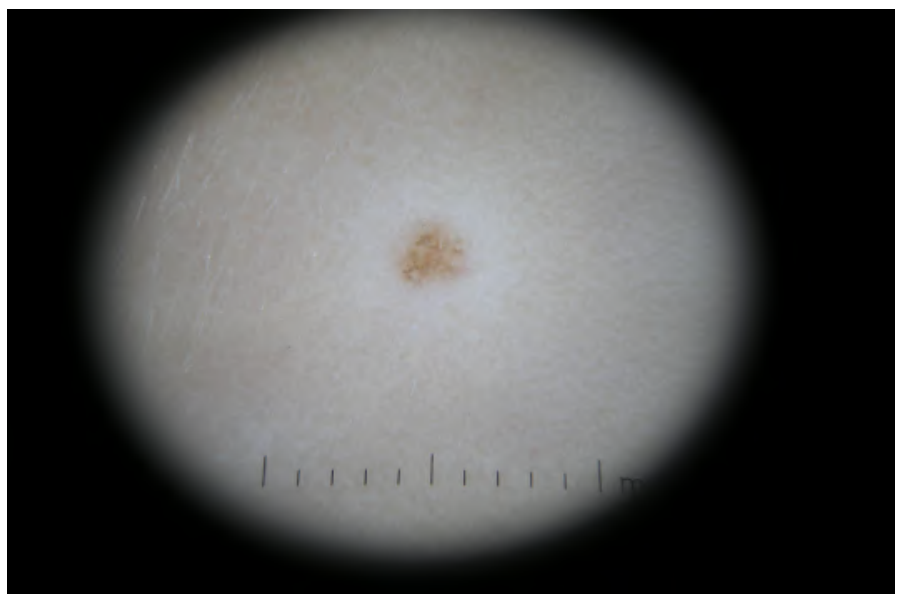

Figure 3: Halo nevus. Dermoscopy $10 \mathrm{x}$ magnification. 


\section{Conclusions}

In a series of 37 halo naevi, ten cases $(27,2 \%)$ had an associated vitiligo, among them $3(33.33 \%)$ had peripheral blood levels of Anti-Thyroid Peroxydase (ATPO). One case from 27 patients with $\mathrm{HN}$ without vitiligo (3.70\%) had high levels of ATPO and ultrasound sign of thyroiditis. In patients with one or several $\mathrm{HN}$, a systematic dermatoscopy and endocrine assessments can reveal associated autoimmune diseases as vitiligo and thyroiditis but halo naevi and vitiligo patients have more frequent high levels of ATPO (33.33\%) than those with just halo naevi without vitiligo $(3.70 \%)$.

\section{Aknowledgement: None.}

\section{References}

1.Sutton, R.L. An unusual variety of vitiligo (leukoderma acquisitum centrifugum). (1916) J Cutan Dis 34: 797-800.

2. Stierman, S.C., Tierney, E.P., Shwayder, T.A. Halo congenital nevocellular nevi associated with extralesional vitiligo: a case series with review of the literature. (2009) Pediatr Dermatol 26(4): 414-424.

Pubmed | Crossref

3. Zeff, R.A., Freitag, A., Grin, C.M., et al. The immune response in halo nevi. (1997) J Am Acad Dermatol 37(4): 620-624.

Pubmed | Crossref

4 . Patrizi, A., Bentivogli, M., Raone, B., et al. Association of halo nevus/i and vitiligo in childhood: a retrospective observational study. (2013) JEADV 27(2): e148-e152.

Pubmed | Crossref | Others

5. Frank, S.B., Cohen, H.J. The halo nevus. (1964) Arch Dermatol 89(3): 367-373.

Others

6. Moretti, S., Spallanzani, A., Pinzi, C., et al. Fibrosis in regressing melanoma versus nonfibrosis in halo nevus upon melanocyte disappearance: could it be related to a different cytokine microenvironment? (2007) J Cutan Pathol 34(4): 301-308.

Pubmed | Crossref | Others

7. de Vijlder, H.C., Westerhof, W., Schreuder, G.M., et al. Difference in pathogenesis between vitiligo vulgaris and halo nevi associated with vitiligo is supported by an HLA association study. (2004) Pigment Cell Res 17(3): 270-274.

Pubmed | Crossref | Others

8. Nedelcu, R.I., Zurac, S.A., Brinzea, A., et al. Morphological features of melanocytic tumors with depigmented halo: review of the literature and personal results. (2015) Rom J Morphol Embryol 56(2 Suppl): 659663.

Pubmed | Others

9. Zabawski, E.J. Jr, Cockerell, C.J. Halo nevus. (2012).

Others

10.Bumbacea, R.S., Popa, L.G., Orzan, O.A., et al. Clinical and therapeutic implications of the association between chronic urticaria and autoimmune thyroiditis. (2014) Acta Endocrinologica (Buc) 10(4): 595-604.

Crossref $\mid$ Others

11. Tatu, A.L., Ionescu, M.A. Multiple autoimmune syndrome type IIIthyroiditis,vitiligo and alopecia areata. (2017) Acta Endo (Buc) 13 (1): 124-125.

12. Tatu, A.L. Topical Steroid Induced Facial Rosaceiform Dermatitis. (2016) Acta Endo (Buc) 12(2): 232-233.

Crossref | Others
Online ISSN: 2381-0858

Journal Title: Investigative Dermatology and Venereology Research Journal Short Name: Invest Dermatol Venereol Res
Ommega Online Publishers

E-mail: editor.dermatology@ommegaonline.org

Website: www.ommegaonline.org 